\title{
Efficacy of Surgery on the Primary Tumour in Patients with Metastatic Bladder Cancer: A Comprehensive Review
}

\author{
Amy H. Lim, Mary E. Westerman, Andrea Korokovic, Justin T. Matulay, \\ Vikram M. Narayan and Neema Navai* \\ Department of Urology, The University of Texas, MD Anderson Cancer Center, Houston, TX, USA
}

Received 22 March 2021

Accepted 19 January 2022

Pre-press 8 February 2022

Published 3 June 2022

\begin{abstract}
.
BACKGROUND: The benefit of surgery of the primary tumor in metastatic bladder cancer is unknown.

OBJECTIVE: Perform a comprehensive contemporary literature review on the benefit of surgery of the primary tumor in metastatic bladder cancer.

METHODS: Ovid MEDLINE, Ovid EMBASE, and Cochrane Library from January 1, 1990 to April 20, 2020 were queried for relevant articles published in English. Each article was evaluated by at least two content experts prior to inclusion which were blinded to the other's evaluation. A third content expert was used when there was not a unanimous decision. Additional articles were added at the discretion of the authors.

RESULTS: Long-term survival is possible in patients with initially unresectable and/or limited metastatic disease. Multimodal therapy with chemotherapy and surgery have the most favorable outcomes when compared to single treatment modalities in selected populations. Patients who demonstrate a robust response to pre-surgical therapy are likely to benefit the most from consolidative surgery. Patients with distant metastatic disease may benefit from consolidative surgery; however, this benefit may only be seen in those with metastatic disease limited to one site.

CONCLUSIONS: Surgery of the primary tumor in metastatic bladder cancer either in the setting of surgery alone, consolidative therapy or coupled with adjuvant therapy may be beneficial in well selected patients and should generally be limited to those who have a response to primary chemotherapy. Randomized clinical control trials are needed to further our understanding of the role of surgery in metastatic bladder cancer.
\end{abstract}

\section{INTRODUCTION}

Bladder cancer is the ninth most common malignancy worldwide [1]. Approximately 5-10\% of patients present with de novo metastatic disease. In

\footnotetext{
*Correspondence to: Neema Navai, MD., Department of Urology, The University of Texas, MD Anderson Cancer Center, 1515 Holcombe Blvd, Unit 1373, Houston, TX 77030, USA. Tel.: +1 713792 3250; Fax: +1 713794 5293; E-mail: nnavai@mdan derson.org.
}

patients with metastatic bladder cancer, cisplatinbased chemotherapy (immunotherapy for those not eligible for cisplatin) and surveillance remain the National Comprehensive Cancer Network's guideline therapy with the inclusion of radical surgery on a select basis in those patients who exhibit a robust clinical response [2]. Despite a response to chemotherapy in $50-60 \%$ of patients, the median survival is estimated to be only 14 months with $14 \%$ survival at 5-years [3] with almost all patients eventually succumbing to cancer related causes. 
In patients with non-metastatic muscle-invasive bladder cancer the outcome is much more favorable with an estimated median survival of $4-6$ years. There is a clear long-term survival benefit and a chance of cure with neoadjuvant cisplatin-based chemotherapy followed by consolidative surgery as demonstrated by randomized control trials $[4,5]$. While guidelines offer consolidative surgery as an option for patients with metastatic bladder cancer with a response to chemotherapy, the evidence demonstrating a survival benefit is not nearly as robust as it is in the setting of non-metastatic disease. However, given the poor outcomes with current systemic treatment options for metastatic bladder cancer and the demonstrated improved survival benefit with chemotherapy and surgical consolidation in non-metastatic muscle invasive disease, it is reasonable to consider and further investigate similar multimodal treatment options for carefully selected patients.

It is difficult to delineate the additional benefit of surgery, if any, compared to chemotherapy alone. Currently, there are no randomized control trials evaluating the benefit of radical cystectomy and pelvic lymph node dissection in patients with metastatic disease. However, there are several retrospective studies that address the question, which are reviewed here. This is a review aimed to provide a comprehensive summary of studies evaluating the survival benefit of consolidative radical cystectomy and pelvic lymph node dissection after chemotherapy in patients with metastatic bladder cancer.

\section{METHODS}

\section{Information sources and search strategy}

We performed a systematic search in Ovid MEDLINE, Ovid EMBASE, and Cochrane Library from January 1, 1990 to April 20, 2020. Search structures, subject headings, and keywords were tailored to each database by a medical research librarian (KJK) specializing in systematic reviews. Searches were restricted to English-only articles and human subjects. Searches were not restricted by study design. We searched multiple grey literature resources such for conferences, dissertations, reports, and other unpublished studies for additional relevant citations. References of the included articles were also searched manually. The search terms used can be found in the complete MEDLINE search strategy which is shown in supplementary Table 1.
Our findings are reported in accordance with the Preferred Reporting Items for Systematic Reviews and Meta-Analyses (PRISMA). Our study protocol is registered in PROSPERO: the International Prospective Register of Systematic Reviews (ID: CRD42020182861).

\section{Study selection}

After the initial search, two of the principal investigators (JTM, AK) independently screened the titles and abstracts of the articles to identify potentially relevant studies. Disagreements were resolved by consensus and by seeking the opinion of a third reviewer (MEW). Studies that passed the title/abstract review were retrieved for full-text review. The two investigators (JTM MEW) then independently screened the remaining full-text articles. Disagreements were resolved by consensus and by seeking the opinion of a third reviewer (AHL). We retrieved 2532 unique articles for review after duplicates were removed. Of these, 21 studies met all the criteria for inclusion in this systematic review (Tables 1-3). The PRISMA flow diagram (Fig. 1) shows the entire review process from the original search to the final selection of studies.

\section{Eligibility criteria}

We included only studies reporting on adult patients ( $>18$ years old) with locally advanced or metastatic urothelial carcinoma of the bladder who underwent radical cystectomy and pelvic lymph node dissection. We excluded review articles, abstracts and case reports. We identified and linked multiple reports of the same study, and we excluded them if duplicated or not relevant. If further clarification was needed regarding reports generated from a potentially overlapping dataset, the authors were contacted. We excluded papers that reported results that had already been published. We included articles that were not identified by the initial search but felt were relevant, which inherently subjects this review to selection bias. No data was combined across multiple studies.

\section{RESULTS}

cT4b/pT4b (Table 1)

Donat et al. performed a retrospective study of 41 patients with $\mathrm{cT} 4 \mathrm{bNx} / \mathrm{N}+\mathrm{M} 0$ disease all of whom were administered methotrexate, vinblastine, dox- 
Table 1

Studies including cT4b/pT4b patients

\begin{tabular}{|c|c|c|c|c|c|c|c|c|c|c|c|c|}
\hline \multirow[t]{3}{*}{ Study } & \multirow[t]{3}{*}{ Pts $(n)$} & \multirow{3}{*}{$\begin{array}{l}\text { Study } \\
\text { description }\end{array}$} & \multirow[t]{3}{*}{ Stage* $^{*}$} & \multicolumn{6}{|c|}{ Chemotherapy/Radiation } & \multirow{3}{*}{$\begin{array}{l}\text { Median } \\
\text { follow up in } \\
\text { months } \\
(\text { range })^{* *}\end{array}$} & \multirow{3}{*}{$\begin{array}{l}\text { Survival/ } \\
\text { recurrence }\end{array}$} & \multirow[t]{3}{*}{ Comments } \\
\hline & & & & \multicolumn{4}{|c|}{ Primary therapy } & \multicolumn{2}{|c|}{ Adjuvant therapy } & & & \\
\hline & & & & Regimen & $\begin{array}{l}\text { Cycles } \\
\text { Median } \\
\text { (range) }\end{array}$ & $\begin{array}{l}\text { Clinical } \\
\text { Response }\end{array}$ & $\begin{array}{l}\text { Pathologic } \\
\text { Response }\end{array}$ & Regimen & $\begin{array}{l}\text { Cycles } \\
\text { Median } \\
\text { (range) }\end{array}$ & & & \\
\hline $\begin{array}{l}\text { Donat, } \\
\text { Herr et al. } \\
1996 \text { [6] }\end{array}$ & $\begin{array}{l}41(24 \\
\text { under- } \\
\text { went } \\
\text { RC) }\end{array}$ & $\begin{array}{l}\text { Retrospective } \\
\text { review of patients } \\
\text { with unresectable } \\
\text { bladder cancer } \\
\text { who received } \\
\text { M-VAC CHT } \\
\text { followed by RC } \\
\text { when possible }\end{array}$ & $\begin{array}{l}\text { cT4bNx/ } \\
+ \text { M0 } \\
\text { cT3-4N+ M0 }\end{array}$ & $\begin{array}{l}100 \% \\
\text { M-VAC }\end{array}$ & $4(2-6)$ & $\begin{array}{l}34 \% \text { cT0 } \\
66 \% \text { cT }+\end{array}$ & $\begin{array}{l}\text { Of the } \\
24 \text { that } \\
\text { under- } \\
\text { went RC, } \\
8 \text { were } \\
\text { pT0 }\end{array}$ & NA & NA & $\begin{array}{l}\text { Minimum } \\
\text { follow up of } 4 \\
\text { years (4-7 } \\
\text { years) }\end{array}$ & $\begin{array}{l}\text { Median survival } \\
\text { for RC ( } 8 \text { ) versus no } \\
\text { RC (6) of } 14 \text { patients } \\
\text { with cCR: } \\
\text { RC: not reached } \\
\text { No RC: } 20 \text { months } \\
\text { Median survival for } \\
\text { RC (16) versus no } \\
\text { RC (11) of } 27 \\
\text { patients with no } \\
\text { cCR: } \\
\text { RC: } 16 \text { months } \\
\text { ( } 2 \text { alive) } \\
\text { No RC: } 14 \text { months } \\
\text { (none alive) }\end{array}$ & $\begin{array}{l}\text { No pelvic tumor recurrences } \\
\text { in } 13 \text { patients, } 9 \text { of which } \\
\text { whom underwent } \\
\text { cystectomy with } 8 \text { alive at } \\
\text { follow up. } \\
\text { Of the } 16 \text { patients with no } \\
\text { clinical response to } \\
\text { M-VAC who underwent } \\
\text { cystectomy, } 7 \text { were down } \\
\text { staged to pT3aN0 or less } \\
\text { and } 2 \text { were alive at follow } \\
\text { up compared to none of the } \\
11 \text { that did not undergo } \\
\text { surgery. Of the } 11 \text { that did } \\
\text { not undergo surgery, } 7 \text { had } \\
\text { inoperable disease, } 4 \text { had } \\
\text { metastatic progression. } \\
\text { Of the } 24 \text { patients who } \\
\text { underwent RC, } 6 \text { ( } 25 \%) \\
\text { required subsequent } \\
\text { exploration and colostomy } \\
\text { to relieve bowel } \\
\text { obstruction due to local } \\
\text { tumor growth. }\end{array}$ \\
\hline $\begin{array}{l}\text { Black, } \\
\text { Dinney } \\
\text { et al. } \\
2011 \text { [7] }\end{array}$ & 23 & $\begin{array}{l}\text { Retrospective } \\
\text { review of patients } \\
\text { with cT4bN0 who } \\
\text { underwent RC }\end{array}$ & $\mathrm{cT} 4 \mathrm{~b}$ & $\begin{array}{l}83 \% \\
\text { M-VAC } \\
\text { Gem/Cis } \\
\text { Taxol based } \\
\text { or some } \\
\text { combination } \\
\text { of the above } \\
13 \% \\
\text { underwent RT } \\
\text { with palliative } \\
\text { intent from } \\
\text { outside } \\
\text { institution }\end{array}$ & NA & NA & $\begin{array}{l}\text { CR: } 28 \% \\
\text { PR: } 35 \%\end{array}$ & $\begin{array}{l}35 \% \\
\text { M-VAC } \\
\text { Gem/Cis } \\
\text { Taxol based } \\
\text { or some } \\
\text { combination } \\
\text { of above }\end{array}$ & NA & $\begin{array}{l}19.2 \\
(6-178.8)\end{array}$ & $\begin{array}{l}1-, 2-\text { and } 5-\text { year } \\
\text { DSS was } 91 \% \\
(95 \% \text { C.I. } 70-98 \%) \\
66 \%(95 \% \text { C.I. } \\
42-83 \%) \text { and } 60 \% \\
(95 \% \text { C.I. } 34-78 \%)\end{array}$ & $\begin{array}{l}\text { Pathologic stage, positive } \\
\text { surgical margin and } \\
\text { presence of lymph node } \\
\text { metastasis at time of } \\
\text { cystectomy correlated with } \\
\text { DSS. } \\
\text { RFS predicted by } \\
\text { pathologic node status (HR } \\
9.72,95 \% \text { CI 1.72-55.1) } \\
\text { and adjuvant chemotherapy } \\
\text { (HR. 5.52, 95\% CI } \\
1.38-22.1 \text { ) using log-rank } \\
\text { test. }\end{array}$ \\
\hline
\end{tabular}



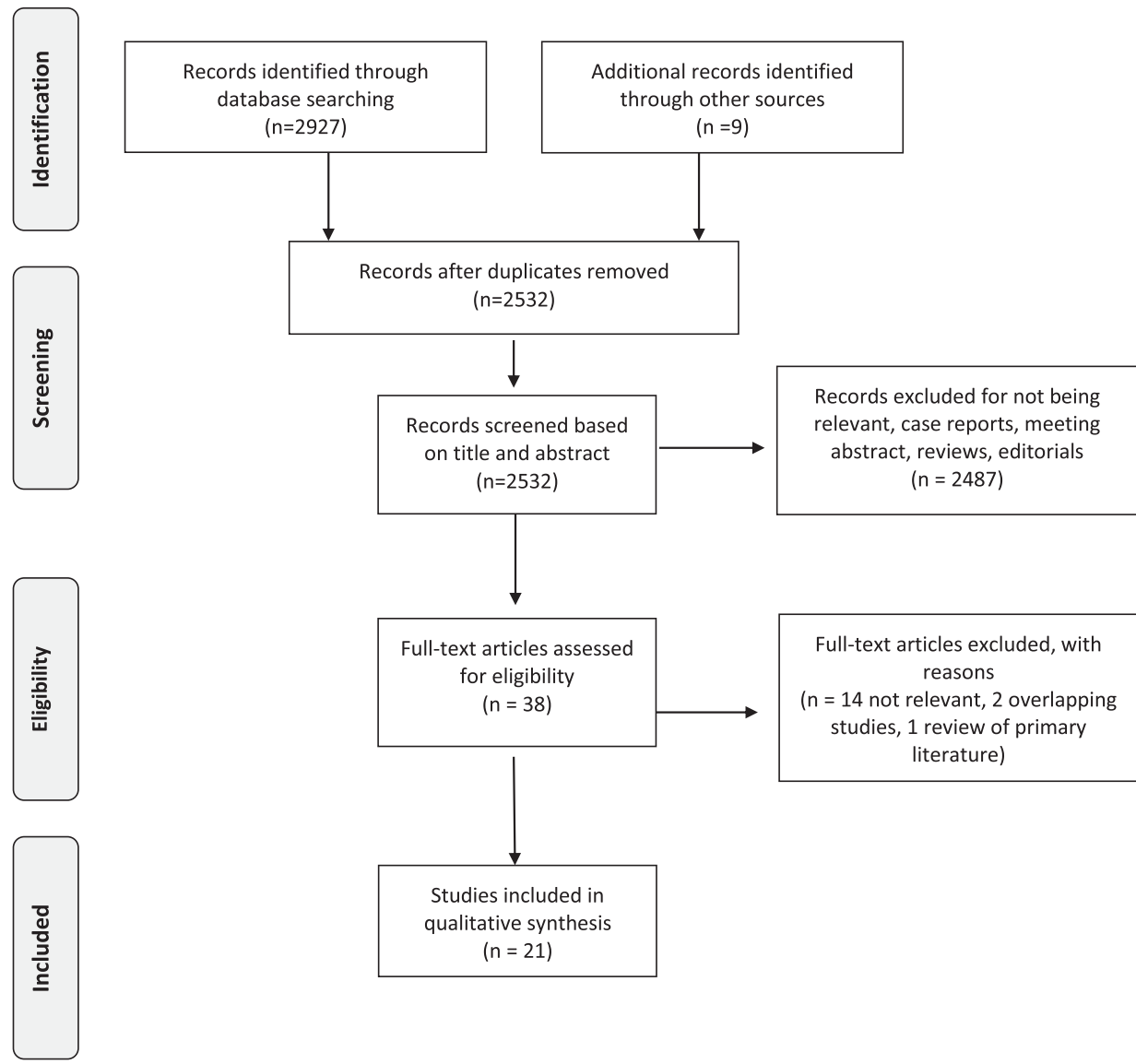

Full-text articles excluded, with reasons

( $n=14$ not relevant, 2 overlapping studies, 1 review of primary literature)

Fig. 1. PRISMA Flow Diagram.

orubicin and cisplatin (M-VAC) as part of M-VAC chemotherapy trials for patients with advanced urothelial cell carcinoma [6]. $23(56 \%)$ of these patients had clinical staging available, $83 \%$ were cT4b. 24 of these patients underwent radical cystectomy. The reasons for not proceeding with surgery were lack of response, systemic tumor progression or patient refusal. $34 \%$ of patients had a complete clinical response. $14(34 \%)$ had a complete clinical response to M-VAC 9 (cT0). 6 of 8 patients who underwent cystectomy compared to only 1 of 5 who refused surgery were alive at 4 years. 7 of the 8 patients who underwent cystectomy were pT0. While these numbers are small, it does demonstrate that despite a complete clinical response in both groups, patients who underwent surgery were more likely to be alive at four years than those who did not undergo surgery. $28(68 \%)$ of the 41 patients had a local recurrence or tumor progression. Not surprisingly, local recurrence or tumor progression occurred in 12 of 14 of patients who had extravesical disease at time of cystectomy, in all of the patients whose cystectomy was aborted due or disease remained inoperable after chemotherapy and in 3 of 5 who refused cystectomy despite having a complete clinical response. On the contrary, only 1 of 10 patients who either had no residual disease in the bladder or had tumor confined to the bladder wall at time of cystectomy had local recurrence or progression of disease. This data suggests that a more robust response to chemotherapy and the ability to achieve negative margins is important in considering which patients will most likely benefit from consolidative surgery.

More recently, Black et al. did a retrospective analysis of patients focusing only on patients with cT4bN0 bladder cancer without evidence of distant metastasis [7]. It was shown that long-term survival was able to be achieved in a portion of patients undergoing primary chemotherapy followed by radical cystectomy. Of the 23 patients identified, $83 \%$ were administered primary chemotherapy and $35 \%$ were administered adjuvant chemotherapy $(13 \%$ underwent palliative radiation prior to surgery or chemotherapy). At time of cystectomy, $17 \%$ were 
pT0 and $8 \%$ had either pTa or pTis and $30 \%$ of patients had pT4 disease. $13 \%$ of patients had positive margins and all died of disease. On univariate analysis, positive surgical margin and pathologic nodal metastasis were predictors of decreased disease survival (HR 5.34, 95\% CI 1.25-22.83 and HR 29.33, 95\% CI 3.13-275.19 respectively).

These studies were small, retrospective, may be favorably skewed due to clinical over staging and the latter only included patients who ultimately had surgery. However, it does highlight that in wellselected patients with initially unresectable disease there can be a robust response to chemotherapy rending consolidative surgery a feasible option. Additionally, in patients with a response to chemotherapy long term disease control and survival is possible following consolidative surgery.

\section{$c N+($ Table 2)}

While the benefit of pre-surgical chemotherapy in patients with muscle-invasive disease has been demonstrated in several studies, historically, few have included patients with clinically node positive disease. However, more recently, retrospective studies have emerged focusing on this patient population. The median survival (overall or cancer specific) ranges from 20-31.6 months [8-11]. Ghadjar et al. did a retrospective study in 30 patients considered unresectable $(21 / 30 \mathrm{cN}+)$ and reported a $30 \%$ pT0 rate and 5-year disease free survival and overall survival of $42 \%$ and $46 \%$ respectively [12].

Galsky et al., utilized the National Cancer Data Base and collected information on 1,739 patients with regionally clinical node positive disease [13]. The crude 5-year survival for chemotherapy alone, cystectomy alone, preoperative chemotherapy followed by surgery and cystectomy followed by adjuvant chemotherapy was $14 \%, 19 \%, 31 \%$ and $26 \%$ respectively. They demonstrated that durable disease-free survival is possible in a subset of patients with clinically node positive disease and that combined therapy with chemotherapy and surgery had the best overall survival compared to surgery alone or chemotherapy alone.

Stanik et al. did a similar study using the Czech National Cancer Registry with similar results [14]. The reported 5-year overall survival rates for chemotherapy alone, radical cystectomy alone and combined radical cystectomy with chemotherapy as $21.7 \%$ (95\% CI $15.4 \%-28.0 \%$ ), $12.1 \%$ (95\% CI $7.4 \%-16.7 \%$ ), and $25.4 \%$ (95\% CI, $18.9 \%-31.9 \%$ ), $p<0.001)$. Similarly, Bae et al., did a retrospective study of 230 patients comparing outcomes based on treatment modalities and also concluded that radical cystectomy in addition to chemotherapy (either before or after surgery) had longer median overall survival compared to chemotherapy or surgery alone (49.1 months, 42.6 months, 19.3 months, 11.2 months respectively, $p<0.001$ ) [15]. Meijer et al., conducted a retrospective study on patients with clinically node positive bladder cancer $(22 \%$ were cM1) who were treated with induction chemotherapy (MVAC or Gem/Cis or Gem/Carbo) followed by surgery [16]. While they determined that prognosis remained poor, $25 \%$ of patients demonstrated a complete pathologic response to primary chemotherapy with a median cancer specific survival of 127 months and 5-year cancer specific survival of $63.5 \%$. Patients with partial, stable or progressive disease after induction chemotherapy had a significantly decreased median survival and 5-year cancer specific survival.

Zargar-Shoshtari et al. performed a large, multiinstitutional retrospective analysis of 304 clinically node positive patients who underwent induction chemotherapy [10]. $85 \%$ of patients underwent platinum-based therapy. The pN0 rate was $56 \%$ in $\mathrm{cN} 1$ patients and $39 \%$ in $\mathrm{cN} 2$ and $\mathrm{cN} 3$ patients $(p=0.03)$ and the pT0 rate was $24 \%$. The pathologic complete response rate for the entire cohort was $14.5 \%$. The median overall survival was 22 months (8.0-54). On multivariate analysis, overall survival was associated with $\mathrm{pN} 0$, negative surgical margins, removal of 15 or more pelvic nodes and cisplatin chemotherapy.

Nieuwenhuijizen et al. did a retrospective review of patients with pathologically confirmed positive lymph nodes either by lymph node dissection or by biopsy treated with chemotherapy alone or with chemotherapy followed by consolidative surgery in the case of partial or complete response [17]. The reported complete response rate was $29 \%$, partial response rate was $57 \%$ and stable or progressive disease was $14 \%$. This study showed also that response to chemotherapy is associated with improved survival and that lymph node status after chemotherapy is critical as all the patients with post chemotherapy nodal disease died within 2 years.

Moschini et al., did a large, single-center retrospective study including 192 patients who did not undergo pre-surgical chemotherapy to evaluate the survival effects of adjuvant chemotherapy in $\mathrm{cN}+$ and $\mathrm{cN}+\mathrm{pN}+$ patients [18]. The $\mathrm{pN}+$ rate was $56 \%$ 
Table 2

Studies including cN+ patients

\begin{tabular}{|c|c|c|c|c|c|c|c|c|c|c|c|c|}
\hline \multirow[t]{3}{*}{$\overline{\text { Study }}$} & \multirow[t]{3}{*}{ Pts $(n)$} & \multirow{3}{*}{$\begin{array}{l}\text { Study } \\
\text { description }\end{array}$} & \multirow[t]{3}{*}{ Stage $^{*}$} & \multicolumn{6}{|c|}{ Chemotherapy/Radiation } & \multirow{3}{*}{$\begin{array}{l}\text { Median follow } \\
\text { up in months } \\
\text { (range) } \text { ( }^{* *}\end{array}$} & \multirow{3}{*}{$\begin{array}{l}\text { Survival/ } \\
\text { recurrence }\end{array}$} & \multirow[t]{3}{*}{ Comments } \\
\hline & & & & \multirow[b]{2}{*}{ Regimen } & \multicolumn{3}{|c|}{ Primary therapy } & \multicolumn{2}{|c|}{ Adjuvant therapy } & & & \\
\hline & & & & & $\begin{array}{l}\text { Cycles } \\
\text { Median } \\
\text { (range) }\end{array}$ & $\begin{array}{l}\text { Clinical } \\
\text { Response }\end{array}$ & $\begin{array}{l}\text { Pathologic } \\
\text { Response }\end{array}$ & Regimen & $\begin{array}{l}\text { Cycles Median } \\
\text { (range) }\end{array}$ & & & \\
\hline $\begin{array}{l}\text { Nieuwen- } \\
\text { huijzen, Bex } \\
\text { et al. 2005 [17] }\end{array}$ & 52 & $\begin{array}{l}\text { Retrospective } \\
\text { review of pN+ } \\
\text { patients treated } \\
\text { with CHT }\end{array}$ & $\begin{array}{l}\text { pN+ by fine } \\
\text { needle bx: } 40 \% \\
\text { pN+ by lym- } \\
\text { phadenectomy: } \\
60 \% \\
\text { pN1: } 21 \% \\
\text { pN2: } 77 \% \\
\text { pN3: } 2 \%\end{array}$ & $\begin{array}{l}\text { HD-M-VAC: } 40 \% \\
\text { M-VAC: } 60 \%\end{array}$ & 4 & $\begin{array}{l}\text { CR: } 29 \% \\
\text { PR: } 57 \% \\
\text { SD/PD: } \\
14 \%\end{array}$ & $\begin{array}{l}\text { In patients with } \\
\text { clinical CR: } \\
73 \% \text { had } \\
\text { complete } \\
\text { pathologic } \\
\text { response } \\
\text { In patients with } \\
\text { clinical PR: } \\
10 \% \text { had } \\
\text { complete } \\
\text { pathologic } \\
\text { response }\end{array}$ & NA & NA & $68(11-105)$ & $\begin{array}{l}5 \text { yr OS: 23\% } \\
\text { (95\% CI 11\%-35\%), } \\
\text { median survival 15.4 } \\
\text { months. } \\
5 \text { yr. } \\
\text { CSS CR: } 42 \% \\
\text { PR: } 19 \% \\
\text { NR: } 0 \%\end{array}$ & $\begin{array}{l}\text { Clinical overall response was } \\
\text { independently associated } \\
\text { with survival (HR. 4.1, } \\
95 \% \text { CI 1.6-10.9) and } \\
\text { more specifically clinical } \\
\text { complete response had } \\
\text { better survival (HR 8.0, } \\
95 \% \text { CI2.4-27.0) } \\
\text { cT0N0 were } \\
\text { independently associated } \\
\text { with improved survival } \\
\text { (HR 4.4, 95\% CI } \\
1.6-12.2) \\
\text { cT+ N0 after M-VAC was } \\
\text { independently related to } \\
\text { better survival (HR 2.8, } \\
95 \% \text { CI 1.1-7.2) }\end{array}$ \\
\hline $\begin{array}{l}\text { Shariat, } \\
\text { Karakiewicz } \\
\text { et al. } 2006 \text { [21] }\end{array}$ & $\begin{array}{l}888(198 \mathrm{pN}+, \\
98 \mathrm{pT} 4)\end{array}$ & $\begin{array}{l}\text { Retrospective } \\
\text { review of } \\
\text { patients } \\
\text { undergoing RC } \\
\text { and BPLND }\end{array}$ & $\begin{array}{l}\text { pN+: } 23 \% \\
\text { pT4: } 12 \%\end{array}$ & $\begin{array}{l}\text { Of entire cohort: } \\
2 \% \text { neoadjuvant } \\
\text { radiation } \\
5 \% \text { upfront } \\
\text { chemotherapy } \\
94 \% \text { with } \\
\text { cisplatin-based } \\
\text { regimens } \\
6 \% \text { carboplatin- } \\
\text { based } \\
\text { regimens }\end{array}$ & NA & NA & $\begin{array}{l}\text { Of entire cohort: } \\
5 \% \text { adj RT } \\
25 \% \text { adj CHT } \\
94 \% \text { with } \\
\text { cisplatin-based } \\
\text { regimens } \\
6 \% \text { carboplatin- } \\
\text { based } \\
\text { regimens }\end{array}$ & NA & NA & $\begin{array}{l}\text { Entire cohort: } \\
39 \text { months } \\
(0.4-183.4)\end{array}$ & $\begin{array}{l}\text { pN- vs pN+ } \\
\text { for } 3,7,10 \text { yr OS: } \\
80.4 \% \text { vs } 37.5 \% \text {, } \\
72.2 \% \text { vs } 27.6 \% \text {, } \\
69.7 \% \text { vs } 25.5 \% \text {. }\end{array}$ & $\begin{array}{l}\text { Patients with pN1-3 were at } \\
\text { significantly higher risk } \\
\text { for bladder cancer } \\
\text { recurrence and death than } \\
\text { patients with pT3NO. }\end{array}$ \\
\hline $\begin{array}{l}\text { Ghadjar, Burkharc } \\
\text { et al. } 2010 \text { [12] }\end{array}$ & & $\begin{array}{l}\text { Retrospective } \\
\text { of consecutive } \\
\text { patients with } \\
\text { unresectable } \\
\text { bladder cancer } \\
\text { who underwent } \\
\text { pre-surgical } \\
\text { CHT followed } \\
\text { by RC }\end{array}$ & $\begin{array}{l}\text { cT4, cN0, cM0: } \\
20 \% \\
\leq \mathrm{cT} 4, \mathrm{cN}+, \\
\text { cM0: } 70 \% \\
\leq \mathrm{cT} 4, \mathrm{cN}+, \\
\text { cM+: } 10 \%\end{array}$ & $\begin{array}{l}\text { Gem/Cis: } 64 \% \\
\text { Gem/Carbo: } \\
\text { 30\% M-VAC: } \\
\text { 3\% } \\
\text { Other: } 3 \%\end{array}$ & $4(2-6)$ & $\begin{array}{l}\text { cN+ patients: } \\
\text { CR: } 76 \% \\
\text { PR: } 5 \% \\
\text { SD: } 19 \%\end{array}$ & $\begin{array}{l}\text { ypT0: } 30 \% \\
\text { ypNo: } 50 \%\end{array}$ & $\begin{array}{l}27 \% \text { underwent } \\
\text { adjuvant } \\
\text { CHT 3\% } \\
\text { underwent } \\
\text { adjuvant } \\
\text { brachyther- } \\
\text { apy }\end{array}$ & NA & $28(4-97)$ & $\begin{array}{l}5 \text { yr DFS: } 42 \% \\
5 \text { yr OS: } 46 \% \\
\text { pT0 patients } \\
5 \text { yr DFS: } 83 \% \\
5 \text { yr OS: } 71 \%\end{array}$ & $\begin{array}{l}\text { pT0 associated with } \\
\text { significant increased DFS } \\
\text { (HR } 0.08,95 \% \text { CI } \\
0.01-0.61 p=0.015 \text { ) and } \\
\text { OS (HR 0.21, 95\% CI } \\
0.05-0.95 \text { ) Age } \leq 64 \\
\text { associated with } \\
\text { significant OS (HR 0.27, } \\
\text { 95\% CI 0.09-0.86), but } \\
\text { not DFS (HR 0.43, 95\% } \\
\text { CI } 0.16-1.19 p=0.1 \text { ) }\end{array}$ \\
\hline
\end{tabular}




\begin{tabular}{|c|c|c|c|c|c|c|c|c|c|c|c|c|}
\hline $\begin{array}{l}\text { Meijer, Mertens } \\
\text { et al. } 2014 \text { [16] }\end{array}$ & 149 & $\begin{array}{l}\text { Retrospective } \\
\text { review of } \mathrm{cN}+ \\
\text { patients treated } \\
\text { with induction } \\
\text { CHT followed } \\
\text { by RC }\end{array}$ & $\begin{array}{l}\mathrm{cN} 1: 36 \% \\
\mathrm{cN} 2-3: 42 \% \\
\mathrm{cM} 1: 22 \% \\
\text { (supra- } \\
\text { regional LN) }\end{array}$ & $\begin{array}{l}\text { M-VAC: } 22 \% \\
\text { HD-M-VAC: } \\
40 \% \\
\text { Gem/Cis: } 13 \% \\
\text { Gem/Carbo: } \\
26 \%\end{array}$ & 4 & $\begin{array}{l}\text { CR: } 35 \% \\
\text { PR: } 48 \% \\
\text { SD: } 12 \% \\
\text { PD: } 5 \%\end{array}$ & $\begin{array}{l}\text { CR: } 27 \% \\
\text { PR: } 28 \% \\
\text { SD: } 24 \% \\
\text { PD: } 10 \% \\
\text { Unknown: } 11 \%\end{array}$ & NA & NA & 57 (24-104) & $\begin{array}{l}\text { Median CSS of cN1: } \\
33 \text { months } \\
\text { cN2-3 and cM1 } 15 \\
\text { months. } \\
5 \text { yr CSS cCR: } 43 \% \\
5 \text { yr CSS pCR: } 64 \%\end{array}$ & $\begin{array}{l}\text { Overall disease recurrence in } \\
\mathrm{cN} 1: 48 \%, \mathrm{cN} 2-3: 64.5 \% \text {, } \\
\mathrm{cM} 1: 61 \% \\
\text { In } \mathrm{cCR} \text {, residual disease } \\
\text { after surgery present } \\
38.5 \% \\
\text { RFS better for } \mathrm{cN} 1 \text { versus } \\
\mathrm{cN} 2-3, \mathrm{cM} 0-1(p=0.03) \\
\mathrm{CSS} \text { of } \mathrm{cN} 2-3 \text { similar to } \\
\mathrm{cM} 1 \text { patients } \\
\text { In cCR, residual disease } \\
\text { present in } 38.5 \% \text { of } \\
\text { patients } \\
\text { pCR associated with CSS } \\
\text { in multi-variate analysis }\end{array}$ \\
\hline $\begin{array}{l}\text { Ho, Willis } \\
\text { et al. 2015[9] }\end{array}$ & 55 & $\begin{array}{l}\text { Retrospective } \\
\text { study } \mathrm{cN}_{+} \\
\text {patients treated } \\
\text { with induction } \\
\text { CHT followed } \\
\text { by RC }\end{array}$ & $\begin{array}{l}\text { cN1: } 29 \% \\
\text { cN2: } 9 \% \\
\text { cN3: } 15 \% \\
\text { M1: } 47 \% \\
\text { (supra- } \\
\text { regional LN) }\end{array}$ & $\begin{array}{l}\text { Cisplatin-based: } \\
93 \% \\
\text { Non-cisplatin } \\
\text { based: } 7 \%\end{array}$ & $5(2-11)$ & $\begin{array}{l}\text { CR: } 38 \% \\
\text { PR: } 46 \% \\
\text { SD: } 9 \% \\
\text { PD: } 2 \% \\
\text { Unknown: } \\
6 \%\end{array}$ & $\begin{array}{l}\text { ypT0N0: } 15 \% \\
\text { ypT+ N0: } 40 \% \\
\text { ypT0N+: } 7 \% \\
\text { ypT+N+: } 38 \%\end{array}$ & NA & NA & $\begin{array}{l}58.7 \\
(0.43-171.8)\end{array}$ & $\begin{array}{l}\text { Overall CSS: } \\
25.7 \text { months } \\
\text { (12.9-NA) } \\
\text { Median CSS in } \\
\text { patients CN1-3: } 22.6 \\
\text { months } \\
\text { 5-year CSS 66\% for } \\
\text { pN0 }\end{array}$ & $\begin{array}{l}\text { No difference in response } \\
\text { rates to CHT between } \\
\text { cN1-3 and cM1 } \\
49 \% \text { had downstaging } \\
\text { after CHT } \\
\text { In cCR, residual disease } \\
\text { present in } 27 \% \\
44 \% \text { of patients with cPR } \\
\text { of nodes were pN0 }\end{array}$ \\
\hline $\begin{array}{l}\text { Urakami, Yuasa } \\
\text { et al. } 2015 \text { [20] }\end{array}$ & 60 & $\begin{array}{l}\text { Retrospective } \\
\text { study of } \mathrm{cN}+ \\
\text { patients treated } \\
\text { with induction } \\
\text { CHT followed } \\
\text { by RC }\end{array}$ & $\begin{array}{l}\text { cN1: } 32 \% \\
\text { cN2: 50\% } \\
\text { cN3: } 18 \% \\
\text { cM1: } 30 \% \\
\text { (supra- } \\
\text { regional LN) } \\
\text { Bladder } \\
\text { primary: 52\% } \\
\text { Upper tract } \\
\text { primary: } 48 \%\end{array}$ & $\begin{array}{l}\text { Gem/Cis based: } \\
57 \% \\
\text { Other: } 43 \%\end{array}$ & $4(2-8)$ & $\begin{array}{l}\text { CR: } 20 \% \\
\text { PR: } 55 \% \\
\text { SD: } 17 \% \\
\text { PD: } 8 \%\end{array}$ & $\begin{array}{l}\text { ypT0: } 20 \% \\
\text { ypN0: } 33 \% \\
\text { ypT0N0: } 14 \% \\
\text { ypTa-1N0: } 8 \% \\
\text { Unknown: } 15 \%\end{array}$ & $\begin{array}{l}69 \% \text { (patients } \\
\text { with pN+, } \\
\text { LVI+, } \\
\text { positive } \\
\text { margin) }\end{array}$ & NA & 22 & $\begin{array}{l}\text { Median PFS: } \\
18.6 \text { months } \\
\text { Median OS: } 31.6 \\
\text { months } \\
5 \text { yr PFS: } 39 \% \\
5 \text { yr PFS CR: } 72 \% \text {, } \\
\text { PR: } 37 \% \text {, SD: } 0 \% \text {. } \\
5 \text { yr OS: } 42 \%\end{array}$ & $\begin{array}{l}16 \% \text { had positive margins } \\
\text { Clinical tumor response } \\
\text { associated with improved } \\
\text { PFS and OS }(p<0.0001)\end{array}$ \\
\hline $\begin{array}{l}\text { Hermans, Fransen } \\
\text { van de Putte } \\
\text { et al. } 2016 \text { [22] }\end{array}$ & 659 & $\begin{array}{l}\text { Retrospective } \\
\text { review of } \mathrm{cN}+ \\
\text { patients treated } \\
\text { with induction } \\
\text { CHT followed } \\
\text { by RC } \\
\text { compared } \\
\text { to RC only }\end{array}$ & $\begin{array}{l}\text { Overall: } \\
\text { cN1: 46\% } \\
\text { cN2-3: 54\% } \\
\text { RC only: } \\
\text { cN1: 60\% } \\
\text { cN2-3: 40\% } \\
\text { primary CHT } \\
\text { and RC: } \\
\text { cN1: } 42 \% \\
\text { cN2-3: 58\% }\end{array}$ & $\begin{array}{l}\text { Overall: } \\
13 \% \text { of } \mathrm{cN} 1 \\
19 \% \text { of } \mathrm{cN} 2-3 \\
\text { Within } \mathrm{CHT} \\
\text { group: } \\
42 \% \text { of } \mathrm{cN} 1 \\
58 \% \text { of } \mathrm{cN} 2-3\end{array}$ & $\mathrm{NA}$ & $\mathrm{NA}$ & $\begin{array}{l}\text { cN1: } \leq \mathrm{ypT} 1 \mathrm{~N} 0 \\
\text { was achieved in } \\
39 \% \\
\text { cN2-3: } \\
\leq \text { ypT1N0 was } \\
\text { achieved in 27\% }\end{array}$ & $\begin{array}{l}8.1 \% \text { of upfront } \\
\text { RC } \\
0.9 \% \text { of RC } \\
\text { and primary } \\
\text { CHT }\end{array}$ & NA & $\begin{array}{l}\text { Upfront RC: } \\
\text { cN1: 73.2 } \\
(37.2-120) \\
\text { cN2-3: } 76.8 \\
(31.2-132) \\
\text { RC and } \\
\text { primary CHT } \\
\text { cN1: 46.8 } \\
(15.6-70) \\
\text { cN2-3: } 31.2 \\
(13.2-78)\end{array}$ & 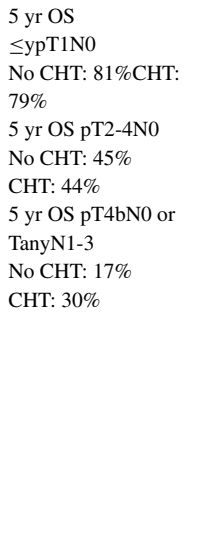 & $\begin{array}{l}\mathrm{pN} 0 \text { at upfront RC cN1: } \\
31 \%, \mathrm{cN} 2-3: 19 \% \\
\mathrm{cN} 1: \leq \mathrm{ypT} \text { T1N0 was } \\
\text { achieved in 39\% } \\
\text { following primary CHT } \\
\text { versus 5\% in RC only } \\
(p<0.001) \\
\text { cN2-3: } \leq \text { ypT1N0 was } \\
\text { achieved in 27\% } \\
\text { following primary CHT } \\
\text { versus 3\% in RC only } \\
\text { ( } p<0.001 \text { ) } \\
\text { primary CHT and RC } \\
\text { associated with } \leq \mathrm{ypT} 1 \mathrm{~N} 0 \\
\text { (OR 14) and 53\% } \\
\text { decreased risk of death } \\
\text { (HR 0.47, 95\% CI } \\
0.36-0.61) \text { compared to } \\
\text { RC alone }\end{array}$ \\
\hline
\end{tabular}


Table 2

\begin{tabular}{|c|c|c|c|c|c|c|c|c|c|c|c|c|}
\hline \multirow[t]{3}{*}{ Study } & \multirow[t]{3}{*}{ Pts $(n)$} & \multirow{3}{*}{$\begin{array}{l}\text { Study } \\
\text { description }\end{array}$} & \multirow[t]{3}{*}{ Stage $^{*}$} & \multicolumn{6}{|c|}{ Chemotherapy/Radiation } & \multirow{3}{*}{$\begin{array}{l}\text { Median follow } \\
\text { up in months } \\
\text { (range)** }\end{array}$} & \multirow{3}{*}{$\begin{array}{l}\text { Survival/ } \\
\text { recurrence }\end{array}$} & \multirow[t]{3}{*}{ Comments } \\
\hline & & & & \multicolumn{4}{|c|}{ Primary therapy } & \multicolumn{2}{|c|}{ Adjuvant therapy } & & & \\
\hline & & & & Regimen & $\begin{array}{l}\text { Cycles } \\
\text { Median } \\
\text { (range) }\end{array}$ & $\begin{array}{l}\text { Clinical } \\
\text { Response }\end{array}$ & $\begin{array}{l}\text { Pathologic } \\
\text { Response }\end{array}$ & Regimen & $\begin{array}{l}\text { Cycles Median } \\
\text { (range) }\end{array}$ & & & \\
\hline $\begin{array}{l}\text { Zargar-Shoshtari, } \\
\text { Zargar et al. } \\
2016[10]\end{array}$ & 304 & $\begin{array}{l}\text { Retrospective } \\
\text { review of } \mathrm{cN}+ \\
\text { patients treated } \\
\text { with induction } \\
\text { chemotherapy } \\
\text { followed by } \\
\text { surgery }\end{array}$ & cN1-3 & $\begin{array}{l}\text { Gem/ } \\
\text { Cis: } 43 \% \\
\text { M-VAC: } 42 \% \\
\text { Other: } 15 \% \\
\text { ( } 85 \% \text { of these } \\
\text { patients } \\
\text { Gem/Carbo) }\end{array}$ & $4(3-4)$ & NA & $\begin{array}{l}\text { ypT0N0: } 15 \% \\
\text { ypT1N0 or } \\
\text { less: } 27 \%\end{array}$ & NA & NA & $13(5-28)$ & $\begin{array}{l}\text { Median OS: } \\
23 \text { months (9-176) } \\
\text { At median of } 10 \\
\text { months: } 50 \% \\
\text { patients died }\end{array}$ & $\begin{array}{l}\text { pT0N0 was only seen in } \\
\text { patients with cN1-N2 } \\
\text { disease } \\
56 \% \mathrm{cN} 1,39 \% \mathrm{cN} 2,39 \% \\
\mathrm{cN} 3 \text { were } \mathrm{pN} 0(p=0.03) \\
\text { Positive margin (HR } 2.96 \text {, } \\
95 \% \mathrm{CI} 1.72-5.09) \text {, single } \\
\text { positive node }(\mathrm{HR}=2.56, \\
95 \% \mathrm{CI} 1.47-4.47), \\
\text { greater than } 2 \text { positive } \\
\text { nodes (HR }=3.26,95 \% \mathrm{CI} \\
1.98-5.36), 15 \text { or greater } \\
\text { nodes removed (0.55, } \\
95 \% \mathrm{Cl} 0.36-0.86) \text { and } \\
\text { receiving M-VAC/GC } \\
\text { (HR=1.88) were } \\
\text { predictors of death. }\end{array}$ \\
\hline $\begin{array}{l}\text { Galsky, Stensland } \\
\text { et al. } 2016 \text { [13] }\end{array}$ & 1739 & $\begin{array}{l}\text { Retrospective } \\
\text { review of } \\
\text { patients in } \\
\text { National Cancer } \\
\text { Database } \\
\text { comparing } \\
\text { outcomes of } \\
\text { patients } \\
\text { undergoing } \\
\text { chemotherapy } \\
\text { and/or radical } \\
\text { cystectomy. }\end{array}$ & $\begin{array}{l}\mathrm{cN} 1: 48 \% \\
\mathrm{cN} 2: 45 \% \\
\mathrm{cN}: 7 \% \\
(1104 \\
\text { underwent } \\
\text { cystectomy and } \\
\text { 635 underwent } \\
\text { CHT alone) }\end{array}$ & $\begin{array}{l}33 \% \text { underwent } \\
\text { primary }\end{array}$ & NA & NA & $\begin{array}{l}\text { ypTON0: } 9 \% \\
\text { ypN0: } 37 \% \\
\text { after CHT } \\
\text { compared to } \\
5 \% \text { undergoing } \\
\text { initial } \\
\text { cystectomy }\end{array}$ & $\begin{array}{l}30 \% \text { underwent } \\
\text { adjuvant } \mathrm{CHT}\end{array}$ & & NA & $\begin{array}{l}5 \text { yr OS for: } \\
\text { CHT alone: } 14 \% \\
\text { Cystectomy } \\
\text { alone: } 19 \% \\
\text { Primary/ } \\
\text { cystectomy: } 31 \% \\
\text { Cystectomy/adj: } \\
: 26 \%\end{array}$ & $\begin{array}{l}\text { Compared with cystectomy } \\
\text { alone, preoperative CHT } \\
\text { associated with } \\
\text { significant improvement } \\
\text { in OS (HR 0.8095\% CI, } \\
0.66 \text { to } 0.97 \text { ). Adjuvant } \\
\text { CHT associated with } \\
\text { improvement in survival } \\
\text { compared to RC alone } \\
\text { (HR 0.68, 95\% CI 0.56 to } \\
0.83 \text { ). Survival of patients } \\
\text { treated with CHT alone } \\
\text { worse than those treated } \\
\text { with cystectomy alone } \\
\text { (HR 1.54, 95\% CI } 95 \% \\
\text { CI, } 1.21 \text { to } 1.96 \text { ) from } 12 \\
\text { months onward. }\end{array}$ \\
\hline $\begin{array}{l}\text { Moschini, Mattei } \\
\text { et al. } 2018 \text { [18] }\end{array}$ & 192 & $\begin{array}{l}\text { Retrospective } \\
\text { review of } \\
\text { patients with } \\
\mathrm{cN}+\text { treated } \\
\text { with RC/PLND } \\
\text { without } \\
\text { pre-surgical } \\
\text { CHT }\end{array}$ & $\begin{array}{l}\mathrm{cN}+: 100 \% \\
\mathrm{pN}+: 56 \% \text { at } \\
\text { time of RC }\end{array}$ & None & NA & NA & NA & $\begin{array}{l}18.8 \% \\
\text { underwent } \\
\text { adjuvant } \mathrm{CHT}\end{array}$ & NA & 48 & $\begin{array}{l}5 \text { yr. RFS: } 55 \% \\
\text { CSM: } 53 \% \\
\text { OM: } 51 \%\end{array}$ & $\begin{array}{l}\text { Adjuvant CHT was } \\
\text { associated with improved } \\
\text { CSS and OS in } \\
\text { multivariate analysis (HR } \\
0.42,95 \% \text { CI } 0.21-0.89 \text {, } \\
p=0.03 \text { and HR: } \\
0.37,95 \% \text { CI } 0.17-0.81)\end{array}$ \\
\hline
\end{tabular}




\begin{tabular}{|c|c|c|c|c|c|c|c|c|c|c|c|c|}
\hline $\begin{array}{l}\text { Stanik, Poprach } \\
\text { et al. } 2019 \text { [14] }\end{array}$ & 661 & $\begin{array}{l}\text { Retrospective } \\
\text { Review of cN+ } \\
\text { patients who } \\
\text { underwent } \\
\text { primary CHT } \\
\text { and RC }\end{array}$ & $\begin{array}{l}\text { cN+ } \\
\text { primary } \\
\text { CHT and } \\
\text { adjuvant CHT } \\
\text { grouped into } \\
\text { "perioperative } \\
\text { CHT" }\end{array}$ & $\begin{array}{l}3 \% \text { under- } \\
\text { went primary } \\
\text { CHT }\end{array}$ & See adj & See adj & See adj & $\begin{array}{l}32 \% \text { underwent } \\
\text { adjuvant CHT }\end{array}$ & NA & NA & $\begin{array}{l}5 \text { yr OS: } \\
\text { RC+CHT no LND: } \\
20.3 \% \\
\text { RC+CHT and LND: } \\
31.9 \% \\
\text { CHT only: } 21.7 \% \\
5 \text { yr CSS: } \\
\text { RC+CHT no LND: } \\
40 \% \\
\text { RC+CHT and LND: } \\
22 \% \\
\text { CHT only: } 30.5 \%\end{array}$ & $\begin{array}{l}5 \text {-year OS rates for } \\
\text { chemotherapy alone, } \\
\text { radical cystectomy alone } \\
\text { and combined radical } \\
\text { cystectomy with } \\
\text { chemotherapy: } p<0.001 \\
\text { Multivariate analysis: } \\
\text { age }>60 \text { years (HR 1.29, } \\
95 \% \text { CI, } 1.06-1.56 \text {; } \\
p=0.011 \text { ), clinical stage } \\
\text { cT3-4 (HR 1.39, } 95 \% \text { CI } \\
1.12-1.71, p=0.002 \text { ) } \\
\text { were negative predictors } \\
\text { of overall survival. } \\
\text { RC+CHT reduced } \\
\text { overall mortality by } 21 \% \\
\text { (HR } 0.79,95 \% \text { CI } \\
0.630-0.994, p=0.044) \\
\text { when compared to CHT } \\
\text { alone. }\end{array}$ \\
\hline $\begin{array}{l}\text { Al-Alao, Mueller- } \\
\text { Leonhard } \\
\text { et al. } 2019 \text { [11] }\end{array}$ & 491 & $\begin{array}{l}\text { Retrospective } \\
\text { review of } \\
\text { patients with } \\
\mathrm{cN}+\text { bladder } \\
\text { cancer treated } \\
\text { with CHT } \\
\text { followed } \\
\text { by RC }\end{array}$ & $\begin{array}{l}\mathrm{cN} 1: 52 \% \\
\mathrm{cN} 2: 42 \% \\
\mathrm{cN} 3: 6 \% \\
\text { All patients } \\
\text { underwent } \\
\text { primary CHT } \\
\text { and RC }\end{array}$ & NA & NA & NA & $\begin{array}{l}10 \% \text { ypT0 } \\
35 \% \text { ypN0 }\end{array}$ & NA & NA & $18.7(8-39)$ & $\begin{array}{l}1,5,8 \text { yr OS: } \\
69 \%, 34 \%, \\
29 \% \\
4 \text { yr OS } \\
\text { cN1: } 22 \% \\
\text { cN2-3: } 12 \% \\
\text { pN0: } 31 \% \\
\text { pN+: } 11 \%\end{array}$ & $\begin{array}{l}\text { On multivariable analysis, } \\
\text { pT3 (HR 2.18 95\% CI } \\
1.31-3.65 ; p=0.003 \text { ) and } \\
\text { pT4 (HR 2.65, 95\% CI } \\
1.53-4.58 ; P<0.001 \text { ) vs } \\
\leq \mathrm{pT} 1 \text { and pN1 (HR 1.77, } \\
95 \% \mathrm{CI} 1.09-2.86 ; \\
p=0.02 \text { ), pN2 (HR } 2.58 \\
95 \% \mathrm{CI} 1.63-4.09 ; \\
p<0.001 \text { ) and pN3 (HR } \\
5.0995 \% \text { CI } 2.55-10.14 \text {; } \\
P<0.001 \text { ) vs pN0 were } \\
\text { independently associated } \\
\text { with worse OS. } \\
5 \text { yr OS: } 10-59 \% \text { of } \\
\text { entire cohort }\end{array}$ \\
\hline $\begin{array}{l}\text { Bae, Lee } \\
\text { et al. } 2019 \text { [15] }\end{array}$ & 230 & $\begin{array}{l}\text { Compare } \\
\text { treatment } \\
\text { outcomes of } \\
\text { CHT before or } \\
\text { after, RC and } \\
\text { RC alone in } \\
\text { patients with } \\
\text { lymph node } \\
\text { positive } \\
\text { bladder } \\
\text { cancer }\end{array}$ & $\begin{array}{l}11.7 \% \text { cystectomy } \\
\text { alone } \\
19.1 \% \text { palliative } \\
\text { CHT } \\
13 \% \text { primary } \\
\text { CHT followed } \\
\text { by RC } \\
56.1 \% \text { RC } \\
\text { followed by adj } \\
\text { CHT } \\
\text { AJC } 7^{\text {th }} \text { criteria: } \\
\text { cN1: } 100 \\
\text { cN2-3: } 130\end{array}$ & $\begin{array}{l}13 \% \text { primary } \\
\text { CHT (all } \\
\text { received a } \\
\text { cisplatin-based } \\
\text { regimen) }\end{array}$ & $2(1-4)$ & NA & NA & $\begin{array}{l}\text { NA56.1\% adj } \\
\text { CHT, } 19.1 \% \\
\text { palliative } \\
\text { CHT } \\
\text { all received } \\
\text { cisplatin- } \\
\text { based } \\
\text { therapy }\end{array}$ & Adj: $3(1-7)$ & $\mathrm{NA}$ & $\begin{array}{l}\text { Median OS for: } \\
\text { entire cohort: } 30.4 \\
\text { months } \\
\text { Palliative CHT: } 19.3 \\
\text { months } \\
\text { Primary CHT/RC: } \\
\text { 49.1 months } \\
\text { RC/Adj CHT: } 42.6 \\
\text { months } \\
\text { RC only: } 11.2 \\
\text { months }\end{array}$ & $\begin{array}{l}\text { The median OS was worse } \\
\text { for } \mathrm{cN} 1 \text { compared to } \mathrm{cN} 2 \\
\text { ( } 42.6 \text { vs } 21.3 \text { months) } \\
p=0.004\end{array}$ \\
\hline
\end{tabular}


reflecting staging inaccuracies problematic to appropriate risk stratification with current imaging techniques and criteria. Multivariable analysis demonstrated that in the $\mathrm{cN}+\mathrm{pN}+$ subgroup, adjuvant chemotherapy was associated with improved cancer specific survival (HR: 0.45, 95\% CI 0.21-0.89, $p=0.03$ ) and overall survival (HR 0.37 , CI $0.17-0.81$, $p=0.01)$. The 5 -year recurrence, cancer specific mortality and overall mortality was $55 \%, 53 \%$ and $51 \%$ with a median follow up of 48 months.

The limitations of these studies are that they are retrospective and two were from cancer registries, which lack granular information such as chemotherapy regimens, comorbidities, performance status, extent of pelvic lymph node dissection, etc. that could bias the results. Additionally, patients that underwent chemotherapy and did not undergo consolidative surgery as a result of having a poor response to chemotherapy may indicate more aggressive disease therefore confounding the results. While these data do not provide level 1 evidence for combination therapy, it provides a compelling argument to consider combination therapy over a single treatment strategy. These retrospective studies varied considerably in the number of patients receiving primary or adjuvant treatment, but nonetheless, they all reiterate the common theme of improved survival in patients undergoing multi-modal therapy. Additionally, the benefit to consolidative surgery is mostly likely in those with complete or partial response to primary chemotherapy resulting in a lower final pathologic stage as suggested in others studies [19-22].

These studies together provide framework that would support the use of a multi-modal approach in patients with metastatic disease who display a measurable response to primary chemotherapy. In the setting of no response or progression, surgery is unlikely to offer a survival benefit and only result in the morbidity of surgery as these patients tend to do poorly regardless of surgical consolidation. However, very few studies have investigated other factors such as quality of life that may be improved from local symptom control from surgery in these patients.

\section{Surgery in patients with extrapelvic lymph node involvement or distant metastasis (cM1) (Table 3)}

While one could consider cystectomy in patients with metastatic disease for palliative purposes, it is difficult to determine what benefit (survival or otherwise), if any, surgery adds to chemotherapy in patients with metastatic disease. It is known that complete response and long-term survival in patients with cisplatin-based chemotherapy is rare and the long-term data regarding treatment with immunetherapy remains to be seen. Therefore, further exploration of whether or not multi-modal therapy can otherwise change this otherwise abruptly lethal disease is warranted.

Several studies have recently emerged comparing effectiveness of treatment modalities in distant metastatic bladder cancer. Seisen et al. conducted a large National Cancer Data Base study evaluating 3,753 patients with metastatic bladder cancer to compare high-intensity treatment of the primary (radical cystectomy or $\geq 50 \mathrm{~Gy}$ delivered to the bladder) to conservative treatment (no surgery, transurethral resection of the bladder or <50Gy of radiation) [23]. The median overall survival was significantly longer in the high-intensity treatment group compared to the conservative group at 14.92 months (9.82-30.72) versus 9.95 months $(5.29-17.08), p<0.001)$. On multivariable analysis, high-intensity local treatment was independently associated with a significant overall survival benefit. While the authors performed a propensity weighted analysis, there were unmeasured confounders such as lack of information regarding adjuvant therapy and burden of disease, body mass index, renal function and cardiovascular disease which may have been prohibited patients from treatment with systemic therapy.

Mao et al. did a retrospective study using the Surveillance, Epidemiology and End Results (SEER) database of patients with stage IV bladder cancer (AJCC $2^{\text {nd }}$ edition- T4bN0M0, TanyN1-3M0, TanyNanyM1) to compare survival based on treatment (chemotherapy, radiation therapy or radical cystectomy) [24]. The median survival for the entire cohort was 9.0 months. Combined chemotherapy and radical cystectomy had a median survival of 18 months and those that received radiation therapy only had a median survival of 3 months which is comparable to those who did not receive any treatment who had a median survival of 2 months. On multivariate analysis, patients who had chemotherapy combined with radical cystectomy was associated with the lowest all-cause mortality (HR $0.26,95 \%$ CI $0.24-0.28, p<0.001)$ and lowest cancer specific mortality (HR $=0.24,95 \%$ CI $0.22-0.26, p<0.001)$. Patients who received radiation therapy only had the highest all-cause mortality $(\mathrm{HR}=0.84,95 \% \mathrm{CI}$ $0.77-0.92, p<0.001)$ and the highest cancer specific mortality (HR $=0.85,95 \%$ CI $0.77-0.94, p=0.002)$. These results demonstrated that combination therapy 
Table 3

Studies including $\mathrm{cM}+$ patients

\begin{tabular}{|c|c|c|c|c|c|c|c|c|c|c|c|c|}
\hline \multirow[t]{2}{*}{ Study } & \multirow[t]{2}{*}{ Pts $(n)$} & \multirow{2}{*}{$\begin{array}{l}\text { Study } \\
\text { description }\end{array}$} & \multirow[t]{2}{*}{ Stage* $^{*}$} & \multicolumn{6}{|c|}{ Chemotherapy/Radiation } & \multirow{2}{*}{$\begin{array}{l}\text { Median } \\
\text { follow up } \\
\text { in months } \\
\text { (range) }{ }^{* *}\end{array}$} & \multirow{2}{*}{$\begin{array}{l}\text { Survival/ } \\
\text { recurrence }\end{array}$} & \multirow[t]{2}{*}{ Comments } \\
\hline & & & & Regimen & $\begin{array}{l}\text { Pr } \\
\text { Cycles } \\
\text { Median } \\
\text { (range) }\end{array}$ & $\begin{array}{l}\text { Cl therapy } \\
\text { Clinical } \\
\text { Response }\end{array}$ & Regimen & \begin{tabular}{l}
\multicolumn{1}{r}{ Adjuvant } \\
$\begin{array}{l}\text { Cycles Median } \\
\text { (range) }\end{array}$
\end{tabular} & $\begin{array}{l}\mathrm{t} \text { therapy } \\
\text { Clinical } \\
\text { Response }\end{array}$ & & & \\
\hline $\begin{array}{l}\text { Seisen, Sun } \\
\text { et al. } 2016 \\
\text { [23] }\end{array}$ & $\begin{array}{l}3753 \\
(248 \\
\text { treated } \\
\text { with RC, } \\
49 \text { treated } \\
\text { with } \\
>50 \mathrm{gy})\end{array}$ & $\begin{array}{l}\text { Retrospective } \\
\text { review using } \\
\text { National } \\
\text { Cancer Data } \\
\text { Base } \\
\text { comparing } \\
\text { CHT with } \\
\text { high-intensity } \\
\text { versus } \\
\text { conservative } \\
\text { treatment of the } \\
\text { primary in } \\
\text { patients with } \\
\text { metastatic } \\
\text { bladder cancer }\end{array}$ & $\begin{array}{l}\text { metastatic bladder } \\
\text { cancer defined } \\
\text { as presence of } \\
\text { extrapelvic } \\
\text { positive lymph } \\
\text { nodes as well as } \\
\text { bone or visceral } \\
\text { involvement } \\
\mathrm{cN}+: 35 \% \\
\text { unknown: } 32 \%\end{array}$ & $\begin{array}{l}\mathrm{r} \text { (CHT given, } \\
\text { information } \\
\text { NA) }\end{array}$ & NA & NA & NA & NA & NA & $\begin{array}{l}64.72(37.59- \\
117.75)\end{array}$ & $\begin{array}{l}\text { Median OS } \\
\text { high-intensity vs } \\
\text { low-intensity: } 14.92 \\
(9.82-30.72) \text { vs } 9.95 \\
(5.29-17.08)\end{array}$ & $\begin{array}{l}\text { High-intensity local therapy was } \\
\text { associated with a significant } \\
\text { overall survival benefit (HR } \\
0.56) \text {. } \\
\text { Median OS significantly } \\
\text { longer in consolidative } \\
\text { surgery group compared to } \\
\text { the cytoreductive strategy } \\
{[17.71(10.41-\mathrm{NA}) \text { vs } 12.42} \\
(7.06-20.37)]\end{array}$ \\
\hline $\begin{array}{l}\text { Li, Kukreja } \\
\text { et al. } 2018 \\
\text { [26] }\end{array}$ & 43 & $\begin{array}{l}\text { Retrospective } \\
\text { review of } \\
\text { patients with } \\
\text { metastatic } \\
\text { bladder cancer } \\
\text { who underwent } \\
\text { cytoreductive } \\
\text { RC }\end{array}$ & $\begin{array}{l}\mathrm{cN}+: 54.6 \% \\
\mathrm{cM}+: 74.4 \%\end{array}$ & $\begin{array}{l}93 \% \text { underwent } \\
\text { primary CHT } \\
51 \% \text { gem/cis } \\
49 \% \text { Adria/gem } \\
46 \% \text { M-VAC }\end{array}$ & $(1-4)$ & $\mathrm{NA}$ & 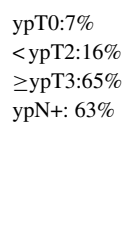 & $\begin{array}{l}16 \% \text { adjuvant } \\
21 \% \text { salvage } \\
16 \% \\
\text { palliative }\end{array}$ & NA & NA & $\begin{array}{l}\text { Median PFS: } \\
5.9 \text { months } \\
\text { Median CSS: } \\
12.3 \text { months }\end{array}$ & $\begin{array}{l}\text { On multivariate analysis, } \\
\text { patients with solitary } \\
\text { metastases had improved CSS } \\
\text { compared to those with } \\
\text { multiple metastases (HR 2.62, } \\
95 \% \text { CI, } 1.16-5.90) \text { with } \\
\text { median CSS of } 26 \text { months } \\
\text { versus } 7.9 \text { months }(p<0.001)\end{array}$ \\
\hline $\begin{array}{l}\text { Luzzago, } \\
\text { Palumbo } \\
\text { et al. } 2019 \\
{[25]}\end{array}$ & 2414 & $\begin{array}{l}\text { Retrospective } \\
\text { review of } \\
\text { SEER database } \\
\text { of patients who } \\
\text { underwent } \\
\text { RC with CHT } \\
\text { vs only CHT in } \\
\text { metastatic } \\
\text { bladder cancer }\end{array}$ & $\begin{array}{l}\text { cNx/N0:52\% } \\
\text { cN1-3:48\% } \\
\text { RC with CHT: } \\
21 \% \\
\text { CHT only: } 79 \%\end{array}$ & NA & $\mathrm{NA}$ & $\mathrm{NA}$ & NA & NA & NA & NA & NA & $\begin{array}{l}\text { Multivariate analysis. RC with } \\
\text { CHT was associated with } \\
\text { lower OM (HR } 0.5,95 \% \text { CI, } \\
0.4-0.6 ; P<.001) \text { after } 1: 1 \mathrm{PS} \\
\text { matching, IPTW and after } \\
\text { accounting for number and } \\
\text { location of metastases. Higher } \\
\text { OS after RC with CHT was } \\
\text { observed in patients with one } \\
\text { metastatic site (21 months vs } \\
6 \text { months, } p=0.001) \text {. }\end{array}$ \\
\hline
\end{tabular}


Table 3

\begin{tabular}{|c|c|c|c|c|c|c|c|c|c|c|c|c|}
\hline \multirow[t]{3}{*}{ Study } & \multirow[t]{3}{*}{ Pts $(n)$} & \multirow{3}{*}{$\begin{array}{l}\text { Study } \\
\text { description }\end{array}$} & \multirow[t]{3}{*}{ Stage* } & \multicolumn{6}{|c|}{ Chemotherapy/Radiation } & \multirow{3}{*}{$\begin{array}{l}\text { Median } \\
\text { follow up in } \\
\text { months } \\
\text { (range) }^{* *}\end{array}$} & \multirow{3}{*}{$\begin{array}{l}\text { Survival/ } \\
\text { recurrence }\end{array}$} & \multirow[t]{3}{*}{ Comments } \\
\hline & & & & \multicolumn{4}{|c|}{ Primary therapy } & \multicolumn{2}{|c|}{ Adjuvant therapy } & & & \\
\hline & & & & Regimen & $\begin{array}{l}\text { Cycles } \\
\text { Median } \\
\text { (range) }\end{array}$ & $\begin{array}{l}\text { Clinical } \\
\text { Response }\end{array}$ & Regimen & $\begin{array}{l}\text { Cycles Median } \\
\text { (range) }\end{array}$ & $\begin{array}{l}\text { Clinical } \\
\text { Response }\end{array}$ & & & \\
\hline $\begin{array}{l}\text { Kim, Park } \\
\text { et al. } 2019 \\
{[33]}\end{array}$ & $\begin{array}{l}130 \\
\text { (88 } \\
\text { bladder, } 42 \\
\text { upper } \\
\text { tract) }\end{array}$ & $\begin{array}{l}\text { Retrospective } \\
\text { review of } \\
\text { patients with } \\
\text { metastatic } \\
\text { bladder cancer } \\
\text { who underwent } \\
\text { CHT+ RC or } \\
\text { CHT only }\end{array}$ & $\begin{array}{l}\text { cM1 patients } \\
\text { with regional } \\
\text { lymph nodes } \\
\text { without distant } \\
\text { metastasis } \\
\text { excluded }\end{array}$ & $\begin{array}{l}\text { Gem/Cis or } \\
\text { carbo } \\
\text { M-VAC }\end{array}$ & NA & NA & $\begin{array}{l}\text { Of RC patients: } \\
16 \% \text { ypT2 } \\
25 \% \text { ypT3 } \\
59 \% \text { ypT4 } \\
19 \% \text { ypN0 } \\
63 \% \text { ypN1 } \\
9 \% \text { ypN2 }\end{array}$ & NA & $\mathrm{NA}$ & $13(6-22)$ & $\begin{array}{l}\text { Median CSS: } \\
16 \text { months in RC/CHT } \\
\text { group, } 10 \text { months in } \\
\text { CHT only. } \\
\text { Median OS: } \\
14 \text { months in RC/CHT } \\
\text { group, } \\
9 \text { months in CHT only }\end{array}$ & $\begin{array}{l}\text { Multivariate analysis } \\
\text { demonstrated higher ECOG } \\
\text { (HR 2.001, 95\% CI } \\
1.313-3.050, p=.001 \text { ) and } \\
\text { metastasis to liver (HR 2.147, } \\
95 \% \mathrm{CI}=1.210-3.807, \\
p=.009) \text { were associated with } \\
\text { worse CSS and OS (HR } \\
2.129,95 \% \mathrm{CI} 1.423-3.185, \\
p<.001) \text { and liver metastasis } \\
(\mathrm{HR}=2.161,95 \% \\
\mathrm{CI}=1.227-3.807, p=.008) . \\
\text { Surgery (HR } 0.663,95 \% \mathrm{CI} \\
0.449-0.980, p=.039) \\
\text { improved CSS in patients } \\
\text { with metastatic UC. Surgery } \\
\text { was not associated with } \\
\text { improved OS. } \\
\text { 20 patients in non-surgery } \\
\text { group required surgical } \\
\text { intervention for urinary tract } \\
\text { related complications } \\
\text { compared to } 1 \text { patient in the } \\
\text { surgery group. }\end{array}$ \\
\hline $\begin{array}{l}\text { Mao, Ma } \\
\text { et al. } 2019 \\
\text { [24] }\end{array}$ & 11824 & $\begin{array}{l}\text { Retrospective } \\
\text { Review of } \\
\text { SEER database } \\
\text { comparing } \\
\text { survival rates of } \\
\text { different } \\
\text { treatment } \\
\text { modalities for } \\
\text { AJCC stage IV } \\
\text { bladder cancer }\end{array}$ & $\begin{array}{l}\text { T4bN0M0, } \\
\text { TanyN1- } \\
\text { 3M0,Tany } \\
\text { NanyM1 } \\
\text { CHT: 52.8\% } \\
\text { RT: } 17 \% \\
\text { RC: } 52.8 \%\end{array}$ & NA & NA & NA & NA & NA & NA & NA & $\begin{array}{l}\text { No CHT, no RT, } \\
\text { No RC: } 2 \text { months } \\
\text { Only CHT: } \\
9 \text { months } \\
\text { Only RT: } \\
3 \text { months } \\
\text { Only RC: } \\
10 \text { months } \\
\text { CHT and RT: } \\
10 \text { months } \\
\text { CHT and RC: } \\
18 \text { months } \\
\text { RT and RC: } \\
8 \text { months } \\
\text { CHT, RT and } \\
\text { RC: } 14 \text { months }\end{array}$ & $\begin{array}{l}\text { Patients who underwent RT } \\
\text { alone had lower OS ( } 14.4 \% \\
\text { vs } 20.5 \%) \\
\text { CHT in combination with RC } \\
\text { had the lowest all-cause } \\
\text { mortality (HR } 0.2695 \% \text { CI } \\
0.24-0.28, p<0.001 \text { ) and } \\
\text { lowest CSS (HR. } 0.24,95 \% \\
\text { CI } 0.22-0.26, p<0.001) \\
\text { Patients who underwent RT } \\
\text { only had the highest all-cause } \\
\text { mortality (HR } 0.84,95 \% \text { CI } \\
0.77-0.92, p<0.001) \text { and the } \\
\text { highest cancer specific } \\
\text { mortality (HR } 0.85,95 \% \text { CI } \\
0.77-0.94, p=0.002) .\end{array}$ \\
\hline
\end{tabular}




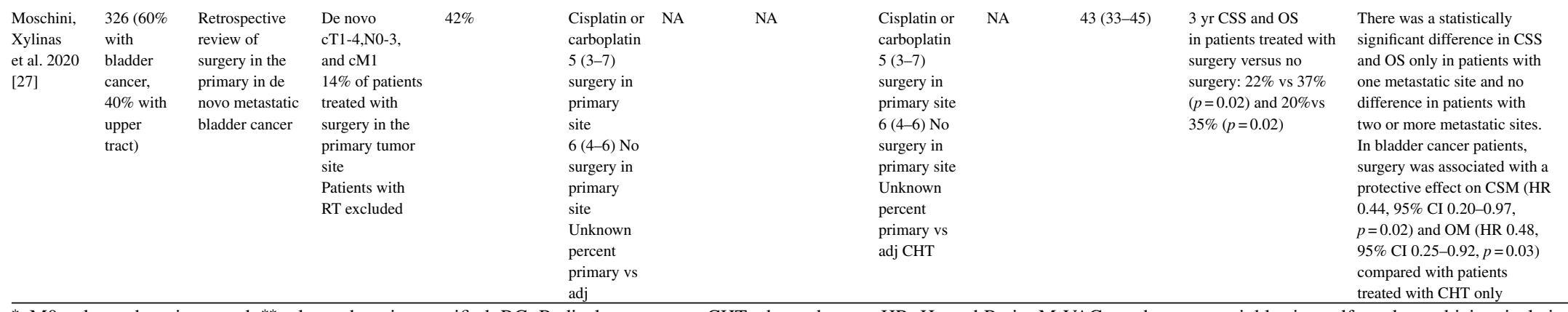

${ }^{*}$ cM0 unless otherwise stated. ${ }^{* *}$ unless otherwise specified. RC: Radical cystectomy. CHT: chemotherapy. HR: Hazard Ratio. M-VAC: methotrexate, vinblastine sulfate, doxorubicin, cisplatin. RFS: recurrence free survival. OS: overall survival. SD: stable disease. PD: progressive disease. CR: complete response. PR: partial response. 
with chemotherapy and radical cystectomy had the best survival rates in patients with metastatic bladder cancer.

Luzzago et al. also did a retrospective study using the SEER registry identifying patients with metastatic urothelial carcinoma of the bladder to determine if there was a difference in survival of patients who underwent chemotherapy alone versus radical cystectomy with chemotherapy [25]. The study included patients from 2004-2016 and on multivariate analysis radical cystectomy with chemotherapy was associated with lower overall mortality before and after propensity score matching (HR $0.5, p<0.001$ and HR $0.5, p<0.001$ respectively). However, higher overall survival after radical cystectomy with chemotherapy was only observed in patients with one metastatic site (21 months vs 16 months, $p=0.001$ ) similar to a study by $\mathrm{Li}$ et al. [26]. It was noted that $78 \%$ of their cohort had one metastatic site and that the number of patients with more than one metastatic site was not robust enough to test whether or not combination therapy was beneficial.

$\mathrm{Kim}$ et al. did a retrospective review comparing patients with metastatic urothelial cancer who underwent chemotherapy with radical cystectomy to chemotherapy only. In this study, which included 130 patients ( 88 bladder, 42 upper tract), the median cancer specific survival and overall survival was 16 months and 14 months in the combination surgery and chemotherapy group compared to 10 months and 9 months, respectively, in the chemotherapy group only $(p=0.014)$. It should be noted that while 20 of 74 patients in the non-surgery group required surgical intervention for urinary tract related complications only 1 of 56 patients in the surgery group required further intervention. The interventions included cystoscopy with fulguration, stent placement, percutaneous nephrostomy tube, and suprapubic tube placement. Although it is important to consider that these procedures are unlikely result in worse morbidity than a cystectomy. They also reported that post-operative complication rate was similar to patients without metastatic disease.

$\mathrm{Li}$ et al. did a retrospective study identifying 43 patients with metastatic urothelial cell carcinoma who underwent radical cystectomy [26]. The median progression free survival was 5.9 months and the median cancer specific survival was 12.3 months. Patients with a single site of metastasis had longer median progression free survival (10.4 months versus 3.5 months) and cancer specific survival (26 months versus 7.9 months) compared to patients with multiple sites of metastasis $(p>0.001)$. On multivariate analysis, solitary metastases compared to those with multiple metastases had improved cancer specific survival (HR 2.62, 95\% CI 1.16-5.90, $p=0.02$ ). There was no difference in survival of patients who had clinically detected versus occult metastasis. The authors noted the 56\% complication rate and $2.3 \%$ mortality rate are similar to the rates in the randomized clinical trial comparing open radical cystectomy to robotic radical cystectomy suggesting the safety of this procedure in this high-risk patient group at high-volume centers.

Similarly, Moschini et al. did a retrospective review of 326 patients of patients with metastatic urothelial carcinoma (60\% with bladder disease, $40 \%$ with upper tract disease) and compared outcomes of those who underwent surgery versus no surgery of the primary site [27]. $14 \%$ of the patient were treated with surgery and patients who underwent radiation therapy were excluded. The 3-year cancer specific and overall survival in patients treated with surgery versus no surgery were $22 \%$ versus $37 \%(p=0.02)$ and $20 \%$ versus $35 \%(p=0.02)$, respectively. There was a statistically significant difference in cancer specific survival and overall survival only in patients with one metastatic site and no difference in patients with two or more metastatic sites.

These studies support further investigation of the role of radical cystectomy after chemotherapy in patients with metastatic bladder cancer. While the studies were retrospective, the majority found that combination therapy resulted in improved overall survival compared to single treatment modalities. Additionally, those with a single site of metastasis had improved survival compared to patients with multiple sites of metastasis when undergoing multimodal therapy. They also demonstrated feasibility and comparable post-operative complication rate when compared to surgery in non-metastatic bladder cancer patients at high volume centers. Importantly, surgery in this patient population may decrease the need for the need for surgical intervention from the morbidity of leaving the bladder in situ. More studies are needed to investigate how surgery may be beneficial to patients with initially metastatic disease outside the parameters of survival such as quality of life.

\section{Limitations and summary of recommendations}

There are several limitations to this review which make it difficult to propose definitive 
recommendations regarding surgery in patients with metastatic disease. Studies were limited to English publications in the Medline database. A validated check list was not used to assess the quality of each of the studies. This review ultimately only included retrospective studies. Additionally, several studies lacked granular data regarding which patients received adjuvant or salvage therapy and the regimen. Some studies did not specify if patients were excluded if they had received pre-surgical or adjuvant radiation. Several of these studies had varying treatment regimens including non-cisplatin-based therapies and a wide range of cycles administered. Additionally, this review spanned four editions of the AJCC Cancer Staging manual, therefore, it is difficult to make specific recommendations about the best prognostic pathologic features as staging has changed throughout the years. Finally, there several articles included in this review were not detected by the original automated search. Therefore, there may be other relevant studies unintentionally not included in our review.

Despite these limitations, there were several recurring themes from the studies that should not be undervalued in addition to several questions raised by the data that should be addressed. First, the patients with metastatic bladder cancer that are most likely to benefit from consolidative surgery are patients who demonstrate a response to chemotherapy. Second, patients with low metastatic burden (limited to a single site) are more likely to have a better outcome with consolidative surgery compared to those with a high metastatic burden. Third, long-term survival is possible in patients with metastatic disease. Fourth, a multi-modal approach (either with surgery or radiation to the primary in addition to chemotherapy) seems to be more beneficial than single modality treatments. Fifth, staging inaccuracies hinder the ability to better risk stratify patients. It is premature to deliver definitive recommendations regarding surgery in patients with metastatic bladder cancer patients as these studies consisted of heterogeneous populations and were subject to the inherent biases of retrospective analyses. However, these studies do form a clear basis for a need for a randomized control trial in this patient population who are at a high risk of death but also maintain a chance for durable disease-free survival.

\section{CONCLUSIONS}

Metastatic bladder cancer has dismal outcomes when treated with chemotherapy alone, however, in select patients, there can be a long-term response with combination therapy. These data support the need for clinical trials addressing the role of surgery in patients with metastatic bladder cancer. It would not be surprising if in the future, the concept of surgery in the primary will also be extended to patients that have received immunotherapy or targeted therapy as there have already been case reports published. Staging continues to be a challenge in bladder cancer and advances in molecular profiling and imaging may change the limitations currently set forth by our current tools.

\section{ACKNOWLEDGMENTS}

We would like to thank the research specialist, Kate Krause (KK) for knowledge and professional guidance throughout this entire process.

\section{FUNDING}

The authors report no funding

\section{AUTHOR CONTRIBUTIONS}

Amy H. Lim, MD, PhD: conception, performance of work, interpretation/analysis of data, writing the article. This author had access to the data. Mary E. Westerman, MD: conception, screening of articles, review of manuscript. This author had access to the data. Andrea Korokovic, MD: conception, screening of articles, review of manuscript. This author had access to the data. Justin T. Matulay, MD: conception, screening of articles. This author had access to the data. Vikram M. Narayan, MD: conception. This author had access to the data. Neema Navai, MD: conception, review of manuscript.

\section{ETHICAL CONSIDERATIONS}

As a literature review, this manuscript is exempt from Institutional Review Board approval. This manuscript did not require the use of animal or human research.

\section{CONFLICT OF INTEREST}

Amy H. Lim, MD, PhD: no conflict of interest to report

Vikram M. Narayan, MD: no conflict of interest to report 
Justin T. Matulay, MD: no conflict of interest to report

Andrea Korokovic, MD: no conflict of interest to report

Mary E. Westerman, MD: no conflict of interest to report

Neema Navai, MD: no conflict of interest to report

\section{SUPPLEMENTARY MATERIAL}

The supplementary material is available in the electronic version of this article: https://dx.doi.org/ 10.3233/BLC-211529.

\section{REFERENCES}

[1] Ploeg M, Aben KK, Kiemeney LA. The present and future burden of urinary bladder cancer in the world. World J Urol. 2009;27(3):289-93.

[2] Network NCCN. Bladder Cancer (Version 6.2020). Available from: https://www.nccn.org/professionals/phy sician_gls/pdf/bladder.pdf.

[3] von der Maase H, Sengelov L, Roberts JT, Ricci S, Dogliotti $\mathrm{L}$, Oliver T, et al. Long-term survival results of a randomized trial comparing gemcitabine plus cisplatin, with methotrexate, vinblastine, doxorubicin, plus cisplatin in patients with bladder cancer. J Clin Oncol. 2005;23(21):4602-8.

[4] Kitamura H, Tsukamoto T, Shibata T, Masumori N, Fujimoto $\mathrm{H}$, Hirao $\mathrm{Y}$, et al. Randomised phase III study of neoadjuvant chemotherapy with methotrexate, doxorubicin, vinblastine and cisplatin followed by radical cystectomy compared with radical cystectomy alone for muscle-invasive bladder cancer: Japan Clinical Oncology Group Study JCOG0209. Ann Oncol. 2014;25(6):1192-8.

[5] Grossman HB, Natale RB, Tangen CM, Speights VO, Vogelzang NJ, Trump DL, et al. Neoadjuvant chemotherapy plus cystectomy compared with cystectomy alone for locally advanced bladder cancer. N Engl J Med. 2003;349(9): 859-66.

[6] Donat SM, Herr HW, Bajorin DF, Fair WR, Sogani PC, Russo P, et al. Methotrexate, vinblastine, doxorubicin and cisplatin chemotherapy and cystectomy for unresectable bladder cancer. The Journal of Urology. 1996;156(2 Pt 1):368-71.

[7] Black PC, Dinney CP, Brown GA, Kassouf W, SiefkerRadtke AO, Munsell MF, et al. The role of radical cystectomy in patients with clinical T4b bladder cancer. Urol Oncol. 2011;29(2):157-61.

[8] Aprikian A, Atef A, Duguid W, Bazinet M, Laplante M, Reid E, et al. Survival after pelvic lymphadenectomy and radical cystectomy in patients with lymph-node metastatic transitional-cell carcinoma of the bladder. Oncol Rep. 1995;2(4):633-5.

[9] Ho PL, Willis DL, Patil J, Xiao L, Williams SB, Melquist $\mathrm{JJ}$, et al. Outcome of patients with clinically node-positive bladder cancer undergoing consolidative surgery after preoperative chemotherapy: The M.D. Anderson Cancer Center Experience. Urologic Oncology. 2015;34(2):59.e1-8.

[10] Zargar-Shoshtari K, Zargar H, Lotan Y, Shah JB, van Rhijn BW, Daneshm, et al. A Multi-Institutional Analysis of Outcomes of Patients with Clinically Node Positive Urothelial
Bladder Cancer Treated with Induction Chemotherapy and Radical Cystectomy. Journal of Urology. 2016;195(1):53-9.

[11] Al-Alao O, Mueller-Leonhard C, Kim SP, Amin A, Tucci $\mathrm{C}$, Kott $\mathrm{O}$, et al. Clinically node-positive $(\mathrm{cN}+)$ urothelial carcinoma of the bladder treated with chemotherapy and radical cystectomy: Clinical outcomes and development of a postoperative risk stratification model. Urologic Oncology. 2019;38(3):76.e19-76.e28.

[12] Ghadjar P, Burkhard FC, Gautschi O, Thalmann GN, Studer UE. Induction chemotherapy for unresectable urothelial carcinoma of the bladder. BJU International. 2010;107(6):894-7.

[13] Galsky MD, Stensland K, Sfakianos JP, Mehrazin R, Diefenbach M, Mohamed N, et al. Comparative Effectiveness of Treatment Strategies for Bladder Cancer With Clinical Evidence of Regional Lymph Node Involvement. J Clin Oncol. 2016;34(22):2627-35.

[14] Stanik M, Poprach A, Zapletalova M, Krejci D, Macik D, Capak I, et al. Comparison of Different Treatment Modalities Outcomes in Clinically Node-positive Bladder Cancer: Analysis of a Population-based Cancer Registry. Clinical Genitourinary Cancer. 2019;17(4):e759-e67.

[15] Bae WK, Lee HJ, Park SH, Kim JH, Kim HJ, Maeng CH, et al. Comparative effectiveness of palliative chemotherapy versus neoadjuvant chemotherapy followed by radical cystectomy versus cystectomy followed by adjuvant chemotherapy versus cystectomy for regional node-positive bladder cancer: A retrospective analysis: KCSG GU 17-03. Cancer Medicine. 2019;8(12):5431-7.

[16] Meijer RP, Mertens LS, van Rhijn BW, Bex A, van der Poel HG, Meinhardt W, et al. Induction chemotherapy followed by surgery in node positive bladder cancer. Urology. 2014;83(1):134-9.

[17] Nieuwenhuijzen JA, Bex A, Meinhardt W, Kerst JM, Schornagel JH, H VANT, et al. Neoadjuvant methotrexate, vinblastine, doxorubicin and cisplatin for histologically proven lymph node positive bladder cancer. The Journal of Urology. 2005;174(1):80-5.

[18] Moschini M, Mattei A, Cornelius J, Shariat SF, Dell'Oglio P, Zaffuto E, et al. Surgical treatment for clinical node-positive bladder cancer patients treated with radical cystectomy without neoadjuvant chemotherapy. World Journal of Urology. 2018;36(4):639-44.

[19] Kassouf W, Agarwal PK, Grossman HB, Leibovici D, Munsell MF, Siefker-Radtke A, et al. Outcome of patients with bladder cancer with $\mathrm{pN}+$ disease after preoperative chemotherapy and radical cystectomy. Urology. 2009; 73(1):147-52.

[20] Urakami S, Yuasa T, Yamamoto S, Sakura M, Tanaka H, Hayashi T, et al. Clinical response to induction chemotherapy predicts improved survival outcome in urothelial carcinoma with clinical lymph nodal metastasis treated by consolidative surgery. Int J Clin Oncol. 2015;20(6): 1171-8.

[21] Shariat SF, Karakiewicz PI, Palapattu GS, Lotan Y, Rogers CG, Amiel GE, et al. Outcomes of radical cystectomy for transitional cell carcinoma of the bladder: a contemporary series from the Bladder Cancer Research Consortium. Journal of Urology. 2006;176(6):2414-22; discussion 22.

[22] Hermans TJ, Fransen van de Putte EE, Horenblas S, Meijer RP, Boormans JL, Aben KK, et al. Pathological downstaging and survival after induction chemotherapy and radical cystectomy for clinically node-positive bladder cancer-Results of a nationwide population-based study. Eur J Cancer. 2016;69:1-8. 
[23] Seisen T, Sun M, Leow JJ, Preston MA, Cole AP, Gelpi-Hammerschmidt F, et al. Efficacy of High-Intensity Local Treatment for Metastatic Urothelial Carcinoma of the Bladder: A Propensity Score-Weighted Analysis From the National Cancer Data Base. J Clin Oncol. 2016;34(29):3529-36.

[24] Mao W, Ma B, Huang X, Gu S, Luo M, Fan J, et al. Which treatment is best for patients with AJCC stage IV bladder cancer? International Urology \& Nephrology. 2019;51(7):1145-56.

[25] Luzzago S, Palumbo C, Rosiello G, Pecoraro A, Deuker M, Tian Z, et al. The effect of radical cystectomy on survival in patients with metastatic urothelial carcinoma of the urinary bladder. Journal of Surgical Oncology. 2019;120(7):126675 .
[26] Li R, Kukreja JEB, Seif MA, Petros FG, Campbell MT, Nguyen JV, et al. The role of metastatic burden in cytoreductive/consolidative radical cystectomy. World Journal of Urology. 2018;37(12):2691-8.

[27] Moschini M, Xylinas E, Zamboni S, Mattei A, Niegisch G, Yu EY, et al. Efficacy of Surgery in the Primary Tumor Site for Metastatic Urothelial Cancer: Analysis of an International, Multicenter, Multidisciplinary Database. Eur Urol Oncol. 2020;3(1):94-101.

[28] Kim J, Park SY, Elghiaty A, Jang WS, Heo JE, Park JS, et al. Is the extirpative surgery for primary tumor helpful for the patients with metastatic urothelial cancer at the time of diagnosis? Medicine (Baltimore). 2019;98(22):e15930. 